\title{
Model of Magnetic Spherical Micro-Robot Motion in Soft Media
}

\author{
Yulia Malkova', Sijie, $\operatorname{Ran}^{2}$, Gary Friedman ${ }^{3}$ \\ ${ }^{1}$ Drexel University \\ 3141 Chestnut St, Philadelphia, PA, USA \\ ym364@drexel.edu; sr923@drexel.edu \\ ${ }^{2}$ Drexel University \\ 3141 Chestnut St, Philadelphia, PA, USA \\ gf29@drexel.edu
}

\section{Extended Abstract}

Millimeter-sized robots are of growing importance for various medical applications involving minimally invasive surgical practices. Such miniature devices could play an important role temporarily during procedures such as laparoscopic and endoscopic techniques or as long-term implants for applications such as drug delivery and neural probes and stimulators. One common difficulty in most of these minimally invasive techniques is insertion of the small device into the target region in the body. In soft tissue insertion, one may often desire to move the device along certain trajectory which may be curved. One method of insertion along curved trajectory may involve pulling the device via magnetic force. However, the movement trajectory depends not only on the applied magnetic force but also on the tissue reaction force. The goal of the presented work is to develop an appropriate model of the tissue reaction force during movement of a small magnetically actuated untethered device in order to plan and control the trajectory of its motion.

There are no macroscopic soft non-linear tissue reaction models, so we would like to propose one. This model takes irreversible energy losses into consideration by presenting medium reaction force or yield force, which depends on the direction of motion with respect to the previous trajectory. By introducing anisotropy direction, the anisotropy force is included in the model. This force plays a major role when solid attempts to translate in the vicinity of the previous pathway, associated with cracks, medium formations, or tissue fracturing around the moving sphere. However, the model ignores any elastic deformations since elastic deformations are small in comparison to sphere position translational changes. By establishing these forces and knowing relations between them, defining a local anisotropy direction as a state variable, it's possible to predict a trajectory of a moving magnetic sphere. Additional focus of the presented work is to test the abovepresented model for different cases and simulate them using MATLAB programming language.

\section{References}

[1] A. Forte, F. Damico, M. Charalambides, D. Dini and J. Williams, "Modelling and experimental characterization of the rate-dependent fracture properties of gelatine gels," Food Hydrocolloids, 46: 180-190, 2015

[2] M. Sitti, H. Ceylan, J. Hu W, Giltinan, M. Turan, S. Yim, and E. Diller, "Biomedical Applications of Untethered Mobile Milli/Microrobots," Proceedings of the IEEE, 103(2): 205-224. URL 10.1109/jproc.2014.2385105, 2015

[3] K. Yesin, K. Vollmers and B. Nelson, "Modeling and Control of Untethered Biomicrorobots in a Fluidic Environment Using Electromagnetic Fields," The International Journal of Robotics Research, 25(5-6): 527-536. 0278364906065389, 2006

[4] S. Ran, "Magnetically Actuated Micro-Robotic Systems for Biomedical Applications," Ph.D. dissertation, Dept. Elect. Eng., Drexel Univ., Philadelphia, PA 